\title{
Citrus flavonoids, b-Glucan and organic acid feed additives decrease relative risk during Yersinia ruckeri 01 biotype 2 infection of rainbow trout (Oncorhynchus mykiss)
}

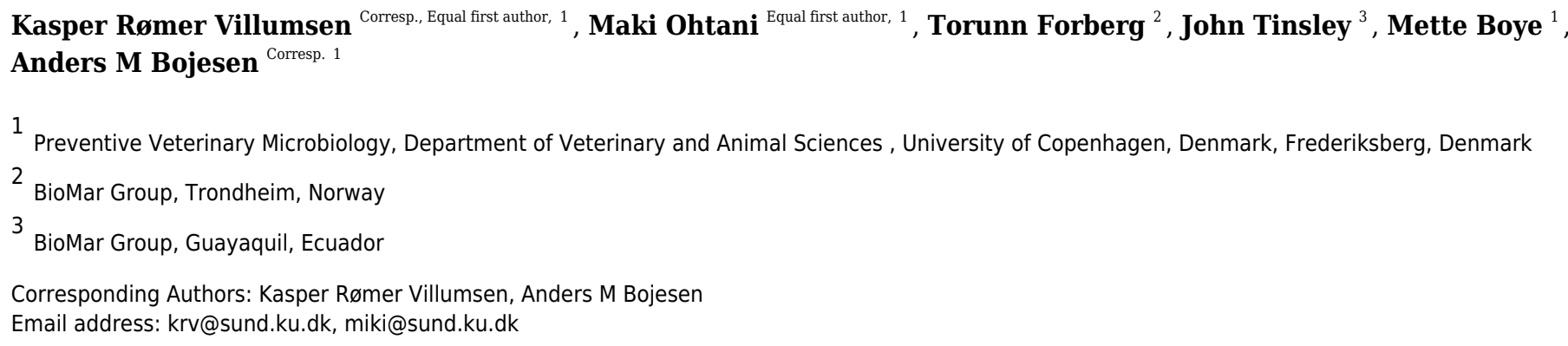

Whether through direct supplementation of bacteria or by prebiotic supplementation thought to favour subsets of bacteria, modulation of gut microbiota constitutes an important and promising alternative to the use of prophylactic and growth promoting antibiotics in worldwide aquaculture. We fed a commercial base feed, alone or supplemented with either proprietary $\beta$-glucan, $\beta$-glucan and organic acids, citrus flavonoid or yeast cell wall supplements, to rainbow trout over a period of four weeks. Fish from each feed group were then subjected to experimental, waterborne infection with Yersinia ruckeri $\mathrm{O} 1$ biotype 2 . Following experimental feeding, the $\beta$-glucan and organic acids supplemented group showed significantly improved feed conversion and lipid efficiency ratios. Furthermore, the b-glucan, $\beta$-glucan and organic acids and citrus flavonoid supplements proved to significantly reduce the risk of mortality in rainbow trout during experimental infection as shown by hazard ratio analysis. Resulting in $33.2 \%$, $30.6 \%$ and $30.5 \%$ reduction in risk relative to the non-supplemented base feed, respectively, these three supplements show a promising potential either as stand-alone feed supplements, or as components in complex feed formulations. 


\section{Citrus Flavonoids, $\beta$-Glucan and Organic Acid Feed}

2 Additives Decrease Relative Risk During Yersinia ruckeri

3 O1 Biotype 2 Infection of Rainbow Trout (Oncorhynchus

4 mykiss)

5

6

7 \& Anders Miki Bojesen ${ }^{1,}$ *

$8{ }^{1}$ Preventive Veterinary Microbiology, Department of Veterinary and Animal Sciences,

9 University of Copenhagen, Denmark

$10{ }^{2}$ BioMar Group, Trondheim, Norway

$11{ }^{3}$ BioMar Group, Guayaquil, Ecuador

12

13

14

15

16

sThese authors have contributed equally to the study.

${ }^{*}$ Corresponding authors:

Kasper Rømer Villumsen: krv@sund.ku.dk

AndersMikiBojesen:miki@sund.ku.dk

Present addresses:

${ }^{\$}$ Division of Development of Functional Brain Activities, Research Centre for Child Mental Development, University of Fukui, Japan.

${ }^{\#}$ Norwegian Veterinary Institute, Norway 


\section{Abstract}

25

26

27

28

29

30

31

32

33

34

35

36

37

38

39

40

41

42

43
Whether through direct supplementation of bacteria or by prebiotic supplementation thought to favour subsets of bacteria, modulation of gut microbiota constitutes an important and promising alternative to the use of prophylactic and growth promoting antibiotics in worldwide aquaculture. We fed a commercial base feed, alone or supplemented with either proprietary $\beta$-glucan, $\beta$ glucan and organic acids, citrus flavonoid or yeast cell wall supplements, to rainbow trout over a period of four weeks. Fish from each feed group were then subjected to experimental, waterborne infection with Yersinia ruckeri $\mathrm{O} 1$ biotype 2. Following experimental feeding, the $\beta$ glucan and organic acids supplemented group showed significantly improved feed conversion and lipid efficiency ratios. Furthermore, the $\beta$-glucan, $\beta$-glucan and organic acids and citrus flavonoid supplements proved to significantly reduce the risk of mortality in rainbow trout during experimental infection as shown by hazard ratio analysis. Resulting in $33.2 \%, 30.6 \%$ and $30.5 \%$ reduction in risk relative to the non-supplemented base feed, respectively, these three supplements show a promising potential either as stand-alone feed supplements, or as components in complex feed formulations.

\section{Introduction}

Intensive rearing practices and continual growth in modern aquaculture places increasing demands on fish feed sourcing, management practices and disease prevention. Along with prophylactic measures such as vaccines, fish feed remains an important field of continual development. As the marine content has been drastically reduced to accommodate the increased demand (Ytrestøyl et al. 2015) and legislative action has been taken against antimicrobial growth promoters, attention has been focused at pre-, pro- and synbiotic feed additives for further optimization (Hoseinifar et al. 2017; Lauzon et al. 2014; Merrifield et al. 2010). While prebiotics are compounds, often oligosaccharides, that are administered in order to promote a beneficial microbial composition (Gibson et al. 2017; Lauzon et al. 2014), probiotics are beneficial bacteria that are directly administered (FAO_WHO 2001; Hill et al. 2014). Finally, synbiotic feed supplements incorporate both pre- and probiotics (Gibson \& Roberfroid 1995). By modulating the composition of the gut microbiota, the aims are to improve feed performance and feed component utilization, but also to improve disease resistance, whether through direct antagonism (Wanka et al. 2018) or competition (Balcázar et al. 2008).

Numerous types of substances have been tested in various aquaculture relevant species, for prebiotic as well as potentially immunomodulating properties, as recently reviewed by Dawood et al. (Dawood et al. 2018) These include yeast cell wall components, typically in the form of various preparations of $\beta$-glucans and mannan oligosaccharides (MOS) (Skov et al. 2012; Staykov et al. 2007; Torrecillas et al. 2014), organic acids and their salts (Gao et al. 2011; Hernández et al. 2012; Yilmaz \& Ergun 2018; Y1lmaz et al. 2019) and citrus flavonoids.

Comprising more than one hundred compounds with antioxidant properties citrus flavonoids are found in Citrus fruits, particular in the peel (Tripoli et al. 2007; Wang et al. 2017), and have 
64 previously been shown to improve resistance towards bacterial infection by intraperitoneal

65

66

67 injection in rainbow trout (Acar et al. 2018).

Previous studies using these feed supplements in various fish species have focused on mitigating adverse effects from antinutritional factors present in certain plant raw materials, growth performance and feed utilization, in some cases followed by experimental or natural infection with either bacterial or parasitic pathogens (Acar et al. 2018; Gao et al. 2011; Hernández et al. 2012; Jaafar et al. 2013; Pandey \& Satoh 2008; Refstie et al. 2010; Staykov et al. 2007; Yilmaz \& Ergun 2018; Y1lmaz et al. 2018; Yu et al. 2014).

Given the importance of the gut homeostasis during bacterial infection (Khimmakthong et al. 2013; Ohtani et al. 2014; Tobback et al. 2010), as well as the hypothesised effects of a modulated gut microbiota, the aim of the present study was to identify prebiotic supplements that will function as stand-alone supplements and/or form the basis of synbiotic feed supplements guided by two focal points: feed performance and resistance towards experimental bacterial infection. An un-supplemented base feed, as well as proprietary experimental feeds supplemented with $\beta$ glucan, $\beta$-glucan combined with organic acids, citrus flavonoids or yeast cell wall extracts, either alone or in combination was fed to rainbow trout fingerlings (Oncorhynchus mykiss). Growth and feed performance parameters were recorded, and finally a waterborne Yersinia ruckeri serotype O1 biotype 2 infection model was employed (Ohtani et al. 2019b). Using an isolate from a Danish freshwater trout farm outbreak, this infection model addresses all natural barriers of the fish, including the gastrointestinal tract, and represents a highly relevant pathogenic threat to commercial rainbow trout farming.

\section{Materials \& Methods}

Ethics statement. The Danish Animal Experiments Inspectorate, under license no. 2015-15-0201-00645 approved the protocols regarding experimental animals described for this study. The study is thus approved under the Danish law regarding experimental animals.

Rainbow trout. Rainbow trout eggs were acquired from AquaSearch Ova (AquaSearch FRESH, Billund, Denmark, 100\% females). Following disinfection with Desamar K30 (Foodtech AG, Uster, Schweiz) according to the manufacturer's instructions, the eggs were hatched and reared under pathogen-free conditions at the Bornholm Salmon Hatchery (Nexø, Denmark). During the rearing period, the fish were fed commercial pelleted feed.

Experimental feeding. Prior to experimental feeding, the fish were transported to the BioMar Research facility (Hirtshals, Denmark), divided into a total of 30 tanks with a volume of 1501 , each holding 29-32 individuals and allowed to acclimatize for 14 days. Once acclimated, each tank was assigned to one of five experimental feed groups resulting in 6 tanks/dietary group. Prior to the initiation of the experimental work, each feed group was anonymized, and the 
104 experiment was performed as a single-blinded setup. This facility has previously been described

105

106

107

108

109

110

111

112

113

114

115

116

117

118

119

120

121

122

123

124

125

126

127

128

129

130

131

132

133

134

135

136

137

138

139

in detail (Ohtani et al. 2019b). In brief, all tanks were supplied through a closed recirculating water system. The water temperature was $14-15^{\circ} \mathrm{C}$, and filtering was performed through physical filtering, as well as a biofilter. The water was oxygenated and passed through an UV-filter (595 $\mu \mathrm{J} / \mathrm{cm}^{2}$ ). At the start of the experimental feeding period, the fish had an average weight of $24.4 \mathrm{~g}$ (bulk weights divided by number of individuals for all tanks).

Each experimental feed type was based on the same proprietary base feed (included as control), and supplemented as described in Table 1 to produce group specific $2 \mathrm{~mm}$ pellets. As all supplemented feeds are proprietary, only general descriptions are given. All pellets were produced by BioMar A/S, and the feeding period was 37 days (1.5\% bodyweight/day).

As a technical error affected waterflow and oxygen supply to one tank in the control feed group, this tank was eliminated from the experiment and downstream analysis.

Experimental feed performance. The performance of each feed group was evaluated based on weight gain (WG), feed conversion ratio (FCR), specific growth rate (SGR), lipid efficiency ratio (LER and protein efficiency ratio (PER). The equations used are given below:

1)

$$
W G=\frac{(\text { Final biomass }(g)-\text { Initial biomass }(g))}{\text { Initial biomass }(g)} \times 100 \%
$$

2)

Total consumed feed $(g)$

$$
F C R=\frac{\text { Total consumed feed }(g)}{(\text { Final biomass }(g)-\text { Initial biomass }(g)+\text { Dead biomass }(g)}
$$

3)

$$
S G R=\frac{(\operatorname{Ln}(\text { Final biomass }(g))-\operatorname{Ln}(\text { Initial biomass }(g))}{\text { Growth period }(\text { days })} \times 100 \%
$$

4)

5)

$$
P E R=\frac{\text { Gained biomass }(g)}{\text { Ingested protein }(g)}
$$

Peer] reviewing PDF | (2019:10:42444:1:2:NEW 22 Jan 2020) 
141 All calculations are made based on tank-wise bulk weighing of total biomass prior to and

142 following the experimental feeding period. Administered feed was recorded for each tank

143 throughout the experimental period and ingested fat/protein is calculated based on total ingested

144 feed and the content of each component in each feed type.

146 Experimental infection. Following the initial feeding period, all individuals from four of the six 147 tanks in each experimental feed group were subjected to waterborne experimental bath infection 148 with Y. ruckeri. The individuals from the remaining two tanks were subjected to mock-infection. 149 Experimental feeding continued throughout the infection period.

150 The bath infection was performed as previously described (Ohtani et al. 2019b). Briefly,

151 cryopreserved Y. ruckeri serotype O1 biotype 2 (strain 07111224) was cultured on blood agar

152 plates for $48 \mathrm{~h}$. Single colonies were then used to inoculate Luria-Bertani broth, which was

153 subsequently incubated at room temperature for $36 \mathrm{~h}$. Prior to infection, the bacteria were

154 harvested by centrifugation and resuspended in clean tank water. Finally, fish from a given tank

155 destined for infection were transferred to a designated infection tank with clean tank water. The

156 infection was then started by the addition of bacterial suspension to a final concentration of

$1577.5 \times 10^{8} \mathrm{CFU} / \mathrm{ml}$. The fish were kept in their respective infection tanks for $3 \mathrm{~h}$, after which they

158 were returned to their holding tanks and monitored closely for 22 days. Mock-infections were

159 performed by transferring fish to separate mock-infection tanks holding clean tank water for $3 \mathrm{~h}$,

160 before returning them to their respective holding tanks, thus mimicking the handling of the

161 infected fish. Experimental feeding continued throughout the infection period, however reduced

162 to $1.1 \%$ biomass/day. During the experimental infection period, all fish that met specified

163 humane endpoints (distinct signs of established disease such as loss of equilibrium, protrusion of

164 either or both eyes, haemorrhages along fin bases) were considered moribund, netted and

165 euthanized with an overdose of benzocaine in accordance with the experimental animal license.

166 The terms "survival", and consequently "mortality", in the following text reflect the binary

167 nature of survival analyses and should therefore be considered technical, rather than purely

168 descriptive terms. Following euthanasia, a swab was made from the anterior kidney onto 5\%

169 blood agar plates to re-isolate the bacterial pathogen in order to satisfy Koch's postulates.

171 Statistical analysis. Throughout the analyses in the present study, a 95\% confidence level was

172 applied using a threshold $\alpha$-level of 0.05 for rejection of the null-hypothesis that there is no

173 difference between the groups in question. All statistical analyses were performed using

174 GraphPad Prism $^{\circledR}$ for Mac (GraphPad, San Diego, USA).

175 Performance data were tested for underlying Gaussian distribution using the Kolmogorov-

176 Smirnov test with Dallal-Wilkinson-Lilliefor correction. Having confirmed this, the means of

177 each experimental group were compared to that of the control feed group using a one-way

178 ANOVA followed by Dunnett's post hoc test multiple comparisons test. 
179 Data from the experimental infection were analysed using the Kaplan-Meier survival analysis 180 tool. Moribund fish from which the bacterial pathogen could be re-isolated were computed as 181 mortalities. In case the pathogen could not be re-isolated, the individual was computed as a 182 censored individual. Based on the course of the survival curves, the log-rank (Mantel-Cox) 183 method was chosen for the analysis. Hazard ratios (log-rank method) were calculated pairwise 184 between all experimental groups, as well. Relative percent survival (RPS) was calculated as 185 follows:

186

187 6)

188

189

Experimental feed performance. Feed performance was determined following the experimental feeding period, and the results are shown in table 2 .

\section{Results}

200 No differences were observed between the control group and any of the experimental groups regarding WG, SGR or PER. The group receiving the $\beta$-glucan + OA supplement was found to have a statistically significantly lower FCR, as well as a higher LER relative to the control group $(\mathrm{P}<0.05)$.

Experimental infection. Mortalities were observed in all infected tanks between 5- and 21-days post infection (Fig. 1). Log-rank survival curve analysis did not identify any statistically significant differences between replicate tanks in any of the groups ( $\mathrm{P}>0.05$, Supplementary Figure S1). Consequently, data from replicate tanks were pooled for each group for all downstream analyses. The results from these analyses are summarized in Fig. 1 and Tables 2 and 3. Group-specific survival curves with $95 \%$ confidence intervals are shown in Supplementary

211 A single mortality occurred from one of the mock-infected tanks during the course of the

212 experimental infection. However, given that only one occurred in a total of six tanks, this is

213 considered negligible.

214 An initial, overall Log-rank survival curve analysis comparing all five survival curves at once

215 did not identify any statistically significant differences between feed groups $(\mathrm{P}=0.13)$. Pairwise

216 comparisons between individual survival curves reached the same overall result, although

217 offering a more nuanced view, as shown in Table 3 . While the $\beta$-glucan, $\beta$-glucan $+\mathrm{OA}$ and $\mathrm{CF}$ 
218 supplemented groups all post P-values $<0.05$ when compared to the control feed group,

219 correction for multiple comparisons reduces the $\alpha$-level to 0.005 . Their respective survival

220

221

222

223

224

225

226

227

228

229

230

231

232

233

234

235

236

237

238

239

240

241

242

243

244

245

246

247

248

249

250

251

252

253

254

255

256

257 curves are therefore not significantly different from the control feed group. Comparisons among the supplemented groups resulted in high P-values $(>0.5)$ indicating similar survival curves in these groups.

When subsequently comparing the relative risk of mortality following infection using the hazard ratios comparing the control group and each of the experimental feed groups in turn, all but the yeast cell wall supplemented feed group displayed hazard ratios with $95 \%$ confidence intervals < 1 (see Fig. 2 and Table 4) These indicate statistically significant reductions in risk following infection relative to the control group (Motulsky 2014).

\section{Discussion}

Following the experimental feeding period, the $\beta$-glucan + OA supplemented feed group showed signs of improved feed utilization when compared to the control feed group, as well as the $\beta$ glucan supplemented feed group. As the study design did not include a group supplemented with organic acids alone, we are unable to assess whether the growth parameters observed for the combined feed is due to the organic acid component or synergies between the $\beta$-glucan and organic acid components. This relationship should be investigated in future studies.

The lowered FCR indicates a significantly increased capacity for converting ingested feed into biomass, while the increased LER suggests that fish in this group utilize the lipid content of the feed in a significantly more efficient manner. A significantly improved utilization of lipid content is noteworthy due to the current issues regarding sourcing of marine content for fish feed (Ytrestøyl et al. 2015). Previous studies have demonstrated a positive effect of either citric or amino acid supplements on retention of phosphorous, SGR and feed utilization in rainbow trout feed (Hernández et al. 2012). While Pandey \& Satoh found that methionine hydroxy analogue supplementation of rainbow trout feed improved the FCR (Pandey \& Satoh 2008), a blend of sodium formate and butyrate was found to improve FCR from fishmeal-based rainbow trout feed (Gao et al. 2011). The same blend, however, was found to decrease the uptake of crude fat from plant-based feed and both effects were found to depend on the extrusion method used. Finally, Yilmaz et al. found that while $0.6 \%$ humic acid sodium salt supplementation improved digestive enzyme activities, this did not translate into changes in FCR or other growth parameters (Y1lmaz et al. 2018). The results from these studies suggests a level of complexity regarding the use of organic acid supplements, most likely reflecting the diversity of this class of supplements. As well as the exact nature of the supplement, dosage is also an important point. Acar et al. demonstrated a dose-dependent effect of pomegranate seed oil (a crude source of the organic punicic acid) supplemented feed on FCR and SGR in rainbow trout (Acar et al. 2018). The results from the experimental Y. ruckeri infection indicated a substantial impact on the experimental groups, leading to a control group endpoint survival of $37.4 \%$. The level of infection obtained is therefore considered sufficient, although rather severe, in terms of providing a realistic platform for evaluating the effects of the experimental prebiotic diets included in the 
258

259

260

261

262

263

264

265

266

267

268

269

270

271

272

273

274

275

276

277

278

279

280

281

282

283

284

285

286

287

288

289

290

291

292

293

294

295

296

297

study. Generally, waterborne infections of more moderate severity would be preferable when evaluating the effect of feed supplements on disease resistance, as stated in our previous studies (Ohtani et al. 2019a). While the initial, overall log-rank survival curve analysis did not identify statistically significant differences between the different feed groups, individual pairwise comparisons showed that the $\beta$-glucan, $\beta$-glucan $+\mathrm{OA}$ and $\mathrm{CF}$ group survival curves differed noticeably, however not statistically significantly, from that of the control group. When estimating the experiment-wide risks calculated for each experimental group relative to that of the control group, the resulting hazard ratios demonstrate statistically significantly reduced risk in the $\beta$-glucan, $\beta$-glucan $+\mathrm{OA}$ and $\mathrm{CF}$ fed groups, relative to that of the control group. While reductions in relative risk observed in this study could be a consequence of the improved performance reported in the present study, the fact that $\beta$-glucans alone, as well as citrus flavonoid supplementation also resulted in significantly reduced relative risk during infection, without the improved feed performance, indicates a more complex background for the observed reductions. While increased fish size has been shown to result in reduced mortalities following bath infection (Ohtani et al. 2019b), the effects of this will be limited within the scope of this study, and supplement specific characteristics will thus play important roles.

The diet including $\beta$-glucan alone reduced the relative risk during infection by $33.2 \%$, with a $95 \% \mathrm{CI}$ that lies well below 1 , and the combination of $\beta$-glucan and organic acids proved to reduce the risk of mortality by $30.6 \%$ relative to that of the control group, also with a $95 \% \mathrm{CI}$ placed below 1 . Previous study results on $\beta$-glucan supplemented feed in rainbow trout have diverged. Skov et al. found no effect of paramylon $(1 \% \mathrm{w} / \mathrm{w})$, a purified $\beta$-glucan product from Euglena gracilis, on rainbow trout resistance towards a waterborne experimental infection with $Y$. ruckeri (Skov et al. 2012). Meanwhile, Ji et al. reported significantly increased endpoint survival following a short intraperitoneal infection with Aeromonas salmonicida in rainbow trout fed $0.05-0.2 \% \beta$-glucan from Saccharomyces cerevisiae. Besides a nearly 30 -fold difference in fish size, these studies vary with respect to pathogen, infection model, statistical analysis and source of $\beta$-glucan, making them hard to compare directly. Our current study design does, however, lie closer to the former. As the potential impact of feed supplements is expected to be less than that of a traditional prophylactic measure, e.g. vaccination, the experimental infection model can prove highly important when assessing their effects (Ohtani et al. 2019a). This, as well as the use of hazard ratio analyses could account for the fact that the current study is able to demonstrate an effect of $\beta$-glucan on the risk of mortality during experimental infection. While the present study did not investigate immune mechanisms, Skov et al. observed an increased expression of lysozyme in $\beta$-glucan fed rainbow trout, however, with no significant increase in lysozyme activity (Skov et al. 2012). Furthermore, Schmitt et al. observed an increased number of cathelicidin producing cells in the proximal intestine of rainbow trout fed $\beta$-glucan (Schmitt et al. 2015), suggesting a role for these bactericidal peptides.

For organic acid supplements, an antimicrobial effect has been suggested through lowering of the $\mathrm{pH}$ of either the host intestinal environment or the cytoplasm of bacteria ( $\mathrm{Ng} \& \mathrm{Koh} 2017)$. This has recently been demonstrated for red hybrid tilapia (Oreochromis sp.) fed feed supplemented 
298

299

300

301

302

303

304

305

306

307

308

309

310

311

312

313

314

315

316

317

318

319

320

321

322

323

324

325

326

327

328

329

330

331

332

333

334

335

336

337

with a proprietary organic acid blend, where inclusion of this blend resulted in significant reduction in gut $\mathrm{pH}$, fecal CFU counts, and significantly reduced mortalities following waterborne infection with Streptococcus agalactiae (Koh et al. 2016). In rainbow trout, studies on trans-cinnamic and humic acid supplements have been shown to result in significantly upregulated innate immune mechanisms including phagocytosis and lysozyme activity from blood samples, that translated into clear and statistically significantly improved survival following intraperitoneal infection with Y. ruckeri (Yilmaz \& Ergun 2018; Y1lmaz et al. 2018). In addition to this, a recent study by Acar et al. investigated the effects of supplementation with pomegranate seed oil containing punicic acid in feed on resistance towards intraperitoneal $Y$. ruckeri infection in rainbow trout, and found a higher, albeit not statistically significant, survival in supplemented groups relative to the control (Acar et al. 2018). The tendency evident from these previous studies support those from the present study. Bypassing mucosal barriers, including the gastrointestinal tract, this suggests a beneficial effect on systemic innate immune mechanisms. Jaafar et al. demonstrated that feed supplemented with a mix of propionic acid, formic acid and silicon dioxide resulted in a shift in allochthonous gut microbiota of rainbow trout, with no apparent effect on disease resistance following low-dose, long-term waterborne infection with Y. ruckeri serotype O1 biotype 2 (Jaafar et al. 2013). In addition to this, feed cosupplementation with Bacillus subtilis and trans-cinnamic acid reportedly boosted the growth of B. subtilis in rainbow trout (Y1lmaz et al. 2019). The potential for modulation of the gut microbiota, as well as the immune system using an organic acid supplement is therefore highly plausible. The co-supplementation with $\beta$-glucan and organic acids in this study, however, does not allow a distinction between their respective contributions.

The citrus flavonoid supplemented feed group showed a $30.5 \%$ reduction in relative risk with a 95\% CI below 1. In a previous study Öntas et al. demonstrated the composition, and antibacterial effect of lemon peel oil against $Y$. ruckeri (ÖntaŞ et al. 2016). While this is a potent source of flavonoids (Wang et al. 2017), it remains a crude mix of multiple components. Specific bacteriostatic and antimicrobial effects of citrus flavonoids have been demonstrated from bergamot (Citrus bergamia Risso) peel extractions (Mandalari et al. 2007). Peel extractions, either native or pectinase treated, as well as purified flavonoids proved mainly effective against Gram-negative bacteria. A study by Vikram et al. indicates that purified citrus flavonoids can act as quorum sensing inhibitors, modulators of bacterial growth and inhibitors of type three secretion system component expression in Vibrio harveyi (Vikram et al. 2010). The presence of a type three secretion system in Y. ruckeri has recently been suggested by Kumar et al. (Kumar et al. 2017), and a combination of general antimicrobial activities and inhibition of pathogenicity could thus be a plausible explanation for the observed reduction in pathogenicity. This would, however, require further studies into the exact molecular effects of the flavonoid supplement in question, and is therefore merely speculative for now.

The yeast cell wall supplement did not confer a significant reduction in relative risk in the present study. Yu et al. (Yu et al. 2014) have previously shown that yeast cell wall extract, including $28 \%$ glucans and $24 \%$ mannan, improved resistance towards intramuscular infection 
338 with Aeromonas veronii in Japanese seabass (Lateolabrax japonicus). While mannan

339 oligosaccharides supplementation has been associated with increased innate immune

340 responsiveness (Staykov et al. 2007) and proposed to limit pathogen establishment through

341 increased mucus production (Torrecillas et al. 2014), and as $\beta$-glucan supplementation has

342 proven successful in this study, as well as in previous studies, crude yeast cell wall extract fails

343 to confer the same reduction in relative risk during infection in the present study.

344

345 Conclusions

$346 \beta$-glucan + OA supplementation resulted in improved feed utilization relative to the base control.

347 Furthermore, $\beta$-glucan, $\beta$-glucan and organic acids, as well as citrus flavonoid supplementation

348 proved to significantly reduce the relative risk of mortality in rainbow trout during experimental

349 infection with $Y$. ruckeri $\mathrm{O} 1$ biotype 2 . These prebiotic and future derived synbiotic feed

350 supplements can therefore be expected to positively affect feed performance and disease

351 resistance in cultured rainbow trout.

352

353 Author contributions

354 Identification of aims and study design: JT, TF, MB \& AMB

355 Execution of experimental work: MO, TF.

356 Data analysis: MO, KRV, TF \& AMB

357 Preparation of manuscript: KRV \& AMB

358 Critical revision and accept of final manuscript: All authors.

359

360 Acknowledgements

361 Anni Nielsen, Miguel Martin and the staff at BioMar Research Facility, Hirtshals, Denmark are

362 acknowledged for their help and expertise throughout the experimental period. 


\section{References}

364 Acar U, Parrino V, Kesbic OS, Lo Paro G, Saoca C, Abbate F, Yilmaz S, and Fazio F. 2018.

365

366

367

368

369

370

371

372

373

374

375

376

377

378

379

380

381

382

383

384

385

386

387

388

389

390

391

392

393

394

395

396

397

398

399

400

401

402

403

404

405

406

407 Effects of Different Levels of Pomegranate Seed Oil on Some Blood Parameters and Disease Resistance Against Yersinia ruckeri in Rainbow Trout. Frontiers in Physiology 9:596. 10.3389/fphys.2018.00596

Balcázar JL, Vendrell D, de Blas I, Ruiz-Zarzuela I, Muzquiz JL, and Girones O. 2008. Characterization of probiotic properties of lactic acid bacteria isolated from intestinal microbiota of fish. Aquaculture 278:188-191.

10.1016/j.aquaculture.2008.03.014

Dawood MAO, Koshio S, and Esteban MÁ. 2018. Beneficial roles of feed additives as immunostimulants in aquaculture: a review. Reviews in Aquaculture 10:950-974. 10.1111/raq.12209

FAO_WHO FaAOotUNaWHO. 2001. Health and Nutritional Properties of Probiotics in Food including Powder Milk with Live Lactic Acid Bacteria.

Gao Y, Storebakken T, Shearer KD, Penn M, and Øverland M. 2011. Supplementation of fishmeal and plant protein-based diets for rainbow trout with a mixture of sodium formate and butyrate. Aquaculture 311:233-240. 10.1016/j.aquaculture.2010.11.048

Gibson GR, Hutkins R, Sanders ME, Prescott SL, Reimer RA, Salminen SJ, Scott K, Stanton C, Swanson KS, Cani PD, Verbeke K, and Reid G. 2017. Expert consensus document: The International Scientific Association for Probiotics and Prebiotics (ISAPP) consensus statement on the definition and scope of prebiotics. Nat Rev Gastroenterol Hepatol 14:491-502. 10.1038/nrgastro.2017.75

Gibson GR, and Roberfroid MB. 1995. Dietary Modulation of the Human Colonic Microbiota - Introducing the Concept of Prebiotics. Journal of Nutrition 125:1401-1412. DOI $10.1093 / \mathrm{jn} / 125.6 .1401$

Hernández AJ, Satoh S, and Kiron V. 2012. Supplementation of Citric Acid and Amino Acid Chelated Trace Elements in Low-Fish Meal Diet for Rainbow Trout Affect Growth and Phosphorus Utilization. Journal of the World Aquaculture Society 43:688-696. doi:10.1111/j.1749-7345.2012.00589.x

Hill C, Guarner F, Reid G, Gibson GR, Merenstein DJ, Pot B, Morelli L, Canani RB, Flint HJ, Salminen S, Calder PC, and Sanders ME. 2014. Expert consensus document. The International Scientific Association for Probiotics and Prebiotics consensus statement on the scope and appropriate use of the term probiotic. Nat Rev Gastroenterol Hepatol 11:506-514. 10.1038/nrgastro.2014.66

Hoseinifar SH, Mirvaghefi A, Amoozegar MA, Merrifield DL, and Ringo E. 2017. In vitro selection of a synbiotic and in vivo evaluation on intestinal microbiota, performance and physiological response of rainbow trout (Oncorhynchus mykiss) fingerlings. Aquaculture Nutrition 23:111-118. 10.1111/anu.12373

Jaafar RM, Kania PW, Larsen AH, Nielsen DS, Fouz B, Browdy C, and Buchmann K. 2013. Gut microbiota changes in rainbow trout, Oncorhynchus mykiss (Walbaum), during organic acid feed supplementation and Yersinia ruckeri infection. J Fish Dis 36:599606. $10.1111 /$ jfd. 12047

Khimmakthong U, Deshmukh S, Chettri JK, Bojesen AM, Kania PW, Dalsgaard I, and Buchmann K. 2013. Tissue specific uptake of inactivated and live Yersinia ruckeri in 
408

409

410

411

412

413

414

415

416

417

418

419

420

421

422

423

424

425

426

427

428

429

430

431

432

433

434

435

436

437

438

439

440

441

442

443

444

445

446

447

448

449

450

451

452

rainbow trout (Oncorhynchus mykiss): Visualization by immunohistochemistry and in situ hybridization. Microbial Pathogenesis 59-60:33-41. DOI 10.1016/j.micpath.2013.03.001

Koh C-B, Romano N, Zahrah AS, and Ng W-K. 2016. Effects of a dietary organic acids blend and oxytetracycline on the growth, nutrient utilization and total cultivable gut microbiota of the red hybrid tilapia, Oreochromis sp., and resistance to Streptococcus agalactiae. Aquaculture Research 47:357-369. doi:10.1111/are.12492

Kumar G, Hummel K, Welch TJ, Razzazi-Fazeli E, and El-Matbouli M. 2017. Global proteomic profiling of Yersinia ruckeri strains. Vet Res 48:55. 10.1186/s13567-017-0460-3

Lauzon HL, Dimitroglou A, Merrifield DL, Ringo E, and Davies SJ. 2014. Probiotics and Prebiotics: Concepts, Definitions and History. Aquaculture Nutrition: Gut Health, Probiotics and Prebiotics, 169-184.

Mandalari G, Bennett RN, Bisignano G, Trombetta D, Saija A, Faulds CB, Gasson MJ, and Narbad A. 2007. Antimicrobial activity of flavonoids extracted from bergamot (Citrus bergamia Risso) peel, a byproduct of the essential oil industry. J Appl Microbiol 103:2056-2064. 10.1111/j.1365-2672.2007.03456.x

Merrifield DL, Harper GM, Dimitroglou A, Ringo E, and Davies SJ. 2010. Possible influence of probiotic adhesion to intestinal mucosa on the activity and morphology of rainbow trout (Oncorhynchus mykiss) enterocytes. Aquaculture Research 41:1268-1272. 10.1111/j.1365-2109.2009.02397.x

Motulsky H. 2014. Intuitive Biostatistics - A Nonmathematical Guide to Statistical Thinking: Oxford University Press.

Ng WK, and Koh CB. 2017. The utilization and mode of action of organic acids in the feeds of cultured aquatic animals. Reviews in Aquaculture 9:342-368. 10.1111/raq.12141

Ohtani M, Villumsen KR, Forberg T, Lauritsen AH, Tinsley J, and Bojesen AM. 2019a. Assessing effects of dietary supplements on resistance against Yersinia ruckeri infection in rainbow trout (Oncorhynchus mykiss) using different infection models. Aquaculture. 10.1016/j.aquaculture.2019.734744

Ohtani M, Villumsen KR, Strøm HK, Lauritsen AH, Aalbæk B, Dalsgaard I, Nowak B, Raida MK, and Bojesen AM. 2019b. Effects of fish size and route of infection on virulence of a Danish Yersinia ruckeri 01 biotype 2 strain in rainbow trout (Oncorhynchus mykiss). Aquaculture 503:519-526. 10.1016/j.aquaculture.2019.01.041

Ohtani M, Villumsen KR, Strøm HK, and Raida MK. 2014. 3D Visualization of the Initial Yersinia ruckeri Infection Route in Rainbow Trout (Oncorhynchus mykiss) by Optical Projection Tomography. PLoS One 9. ARTN e89672

10.1371/journal.pone.0089672

Pandey A, and Satoh S. 2008. Effects of organic acids on growth and phosphorus utilization in rainbow trout Oncorhynchus mykiss. Fisheries Science 74:867-874. 10.1111/j.1444-2906.2008.01601.x

Refstie S, Baeverfjord G, Seim RR, and Elvebø 0. 2010. Effects of dietary yeast cell wall betaglucans and MOS on performance, gut health, and salmon lice resistance in Atlantic salmon (Salmo salar) fed sunflower and soybean meal. Aquaculture 305:109-116. 10.1016/j.aquaculture.2010.04.005

Schmitt P, Wacyk J, Morales-Lange B, Rojas V, Guzman F, Dixon B, and Mercado L. 2015. Immunomodulatory effect of cathelicidins in response to a beta-glucan in intestinal 
453

454

455

456

457

458

459

460

461

462

463

464

465

466

467

468

469

470

471

472

473

474

475

476

477

478

479

480

481

482

483

484

485

486

487

488

489

490

491

492

493

494

495

496

epithelial cells from rainbow trout. Dev Comp Immunol 51:160-169.

10.1016/j.dci.2015.03.007

Skov J, Kania PW, Holten-Andersen L, Fouz B, and Buchmann K. 2012. Immunomodulatory effects of dietary beta-1,3-glucan from Euglena gracilis in rainbow trout (Oncorhynchus mykiss) immersion vaccinated against Yersinia ruckeri. Fish \& Shellfish Immunology 33:111-120. 10.1016/j.fsi.2012.04.009

Staykov Y, Spring P, Denev S, and Sweetman J. 2007. Effect of a mannan oligosaccharide on the growth performance and immune status of rainbow trout (Oncorhynchus mykiss). Aquaculture International 15:153-161. 10.1007/s10499-007-9096-z

Tobback E, Hermans K, Decostere A, Van den Broeck W, Haesebrouck F, and Chiers K. 2010. Interactions of virulent and avirulent Yersinia ruckeri strains with isolated gill arches and intestinal explants of rainbow trout Oncorhynchus mykiss. Dis Aquat Organ 90:175-179.

Torrecillas S, Montero D, and Izquierdo M. 2014. Improved health and growth of fish fed mannan oligosaccharides: Potential mode of action. Fish \& Shellfish Immunology 36:525-544. 10.1016/j.fsi.2013.12.029

Tripoli E, La Guardia M, Giammanco S, Di Majo D, and Giammanco M. 2007. Citrus flavonoids: Molecular structure, biological activity and nutritional properties: A review. Food Chemistry 104:466-479. 10.1016/j.foodchem.2006.11.054

Vikram A, Jayaprakasha GK, Jesudhasan PR, Pillai SD, and Patil BS. 2010. Suppression of bacterial cell-cell signalling, biofilm formation and type III secretion system by citrus flavonoids. J Appl Microbiol 109:515-527. 10.1111/j.1365-2672.2010.04677.x

Wang SC, Yang CK, Tu H, Zhou JJ, Liu XQ, Cheng YJ, Luo J, Deng XX, Zhang HY, and Xu J. 2017. Characterization and Metabolic Diversity of Flavonoids in Citrus Species. Scientific Reports 7. ARTN 10549

10.1038/s41598-017-10970-2

Wanka KM, Damerau T, Costas B, Krueger A, Schulz C, and Wuertz S. 2018. Isolation and characterization of native probiotics for fish farming. Bmc Microbiology 18:119. 10.1186/s12866-018-1260-2

Yilmaz S, and Ergun S. 2018. Trans-cinnamic acid application for rainbow trout (Oncorhynchus mykiss): I. Effects on haematological, serum biochemical, nonspecific immune and head kidney gene expression responses. Fish Shellfish Immunol 78:140-157. 10.1016/j.fsi.2018.04.034

Yılmaz S, Ergun S, Çelik EŞ, and Yigit M. 2018. Effects of dietary humic acid on growth performance, haemato-immunological and physiological responses and resistance of Rainbow trout, Oncorhynchus mykiss

to Yersinia ruckeri. Aquaculture Research 49:3338-3349. 10.1111/are.13798

Yılmaz S, Ergun S, Yigit M, and Çelik EŞ. 2019. Effect of combination of dietary Bacillus subtilis and trans - cinnamic acid on innate immune responses and resistance of rainbow trout, Oncorhynchus mykiss to Yersinia ruckeri. Aquaculture Research. 10.1111/are.14379

Ytrestøyl T, Aas TS, and Åsgård T. 2015. Utilisation of feed resources in production of Atlantic salmon (Salmo salar) in Norway. Aquaculture 448:365-374.

10.1016/j.aquaculture.2015.06.023

Peer] reviewing PDF | (2019:10:42444:1:2:NEW 22 Jan 2020) 
497 Yu HH, Han F, Xue M, Wang J, Tacon P, Zheng YH, Wu XF, and Zhang YJ. 2014. Efficacy and 498 tolerance of yeast cell wall as an immunostimulant in the diet of Japanese seabass 499

500 (Lateolabrax japonicus). Aquaculture 432:217-224.

501 ÖntaȘ C, Baba E, Kaplaner E, Kücükaydin S, Öztürk M, and Ercan MD. 2016. Balık 502

503

504 on) ve Argan (Argania spinosa) Yağının

505 Antibakteriyel Aktivitesi. Kafkas Universitesi Veteriner Fakultesi Dergisi. 10.9775/kvfd.2016.15311 


\section{Table $\mathbf{1}$ (on next page)}

Composition of each feed group used in the experimental setup.

Composition of each feed group used in the experimental setup. 
$1 \quad$ Table 1

2

\begin{tabular}{|c|c|}
\hline Group designation: & Content: \\
\hline Control - Base feed & $\begin{array}{l}48 \% \text { protein, } 23 \% \text { fat, } 20 \mathrm{MJ} / \mathrm{kg}, 35 \% \text { marine content, } 26 \% \\
\text { fishmeal }\end{array}$ \\
\hline$\beta$-glucan & $\begin{array}{l}\text { Base feed supplemented with } \beta \text {-glucans, vitamins } \mathrm{C}+\mathrm{E} \text { and } \\
\text { nucleotides }\end{array}$ \\
\hline$\beta$-glucan + OA & $\begin{array}{l}\text { Base feed supplemented with } \beta \text {-glucans and organic acids, } \\
\text { vitamins } \mathrm{C}+\mathrm{E} \text { and nucleotides }\end{array}$ \\
\hline $\mathrm{CF}$ & $\begin{array}{l}\text { Base feed supplemented with citrus flavonoids, vitamins } \mathrm{C}+\mathrm{E} \\
\text { and nucleotides }\end{array}$ \\
\hline YCW & $\begin{array}{l}\text { Base feed supplemented with yeast cell wall extract vitamins } \mathrm{C}+ \\
\text { E and nucleotides }\end{array}$ \\
\hline
\end{tabular}

Table 1: Composition of each feed group used in the experimental setup. 


\section{Table 2 (on next page)}

Feed performance data following experimental feeding

Weight gain (WG), feed conversion ratio (FCR), specific growth rate (SGR), lipid efficiency ratio (LER) and protein efficiency ratio (PER) data (mean \pm standard deviation) for the experimental feeding period. All calculations were performed as described above. Asterisks denote significant difference from control group values $(P<0.05)$. 
1 Table 2

2

\begin{tabular}{|c|c|c|c|c|c|}
\hline & Control & $\beta$-glucan & $\begin{array}{c}\beta \text {-glucan }+ \\
O A\end{array}$ & $C F$ & $Y C W$ \\
\hline$W G$ & $116.8( \pm 3.2)$ & $120.4( \pm 7.1)$ & $\begin{array}{l}119.1 \\
( \pm 3.3)\end{array}$ & $\begin{array}{l}119.2 \\
( \pm 3.6)\end{array}$ & $\begin{array}{l}114.9 \\
( \pm 8.6)\end{array}$ \\
\hline$F C R$ & $0.68( \pm 0.04)$ & $0.66( \pm 0.03)$ & $\begin{array}{l}0.60 \\
( \pm 0.01) *\end{array}$ & $\begin{array}{l}0.63 \\
( \pm 0.02)\end{array}$ & $\begin{array}{l}0.64 \\
( \pm 0.07)\end{array}$ \\
\hline$S G R(\%)$ & $2.11( \pm 0.03)$ & $2.16( \pm 0.11)$ & $\begin{array}{l}2.14 \\
( \pm 0.05)\end{array}$ & $\begin{array}{l}2.14 \\
( \pm 0.07)\end{array}$ & $\begin{array}{l}2.08 \\
( \pm 0.12)\end{array}$ \\
\hline$L E R$ & $6.57( \pm 0.37)$ & $6.91( \pm 0.29)$ & $\begin{array}{l}7.46 \\
( \pm 0.16) *\end{array}$ & $\begin{array}{l}7.04 \\
( \pm 0.22)\end{array}$ & $\begin{array}{l}7.18 \\
( \pm 0.84)\end{array}$ \\
\hline PER & $3.10( \pm 0.17)$ & $3.23( \pm 0.13)$ & $\begin{array}{l}3.41 \\
( \pm 0.07)\end{array}$ & $\begin{array}{l}3.26 \\
( \pm 0.10)\end{array}$ & $\begin{array}{l}3.30 \\
( \pm 0.39)\end{array}$ \\
\hline
\end{tabular}

3 Table 2: Weight gain (WG), feed conversion ratio (FCR), specific growth rate (SGR), lipid

4 efficiency ratio (LER) and protein efficiency ratio (PER) data (mean \pm standard deviation) for

5 the experimental feeding period. All calculations were performed as described above. Asterisks

6 denote significant difference from control group values $(P<0.05)$.

7 


\section{Table 3(on next page)}

$P$-values from pairwise log-rank survival curve analyses.

$P$-values from pairwise log-rank survival curve analyses. The Bonferroni-corrected a-level is 0.005 . 


\section{$1 \quad$ Table 3}

2

\begin{tabular}{l|ccccc}
\hline & Control & $\beta$-glucan & $\begin{array}{c}\beta \text {-glucan }+ \\
\text { OA }\end{array}$ & CF & YCW \\
\hline Control & - & 0.021 & 0.039 & 0.038 & 0.085 \\
$\beta$-glucan & & - & 0.886 & 0.890 & 0.567 \\
$\beta$-glucan + OA & & - & 0.987 & 0.705 \\
CF & & & - & 0.712 \\
YCW & & & & & - \\
\hline
\end{tabular}

3 Table 3: P-values from pairwise log-rank survival curve analyses. The Bonferroni-corrected $\alpha$ 4 level is 0.005. 


\section{Table 4 (on next page)}

Results from experimental infection

Results from the experimental infection. All hazard ratios are relative to the control group. See Materials \& Methods for details. 
1 Table 4

2

\begin{tabular}{llll}
\hline Group: & Endpoint survival: & RPS: & Hazard ratio $(95 \%$ CI): \\
\hline Control & $37.4 \%$ & - & - \\
$\beta$-glucan & $48.8 \%$ & $18.2 \%$ & $0.6677(0.4385-0.9192)$ \\
$\beta$-glucan + OA & $50.0 \%$ & $20.2 \%$ & $0.6937(0.4591-0.9647)$ \\
CF & $48.8 \%$ & $18.2 \%$ & $0.6946(0.4605-0.9622)$ \\
YCW & $48.3 \%$ & $17.5 \%$ & $0.7400(0.4952-1.030)$ \\
\hline
\end{tabular}

3 Table 4: Results from the experimental infection. All hazard ratios are relative to the control

4 group. See Materials \& Methods for details. 
Figure 1

Kaplan-Meier survival curves

Kaplan-Meier survival curve analysis (Log-rank) of pooled replicates for each feed group.

Statistical analysis is explained in Materials \& Methods.

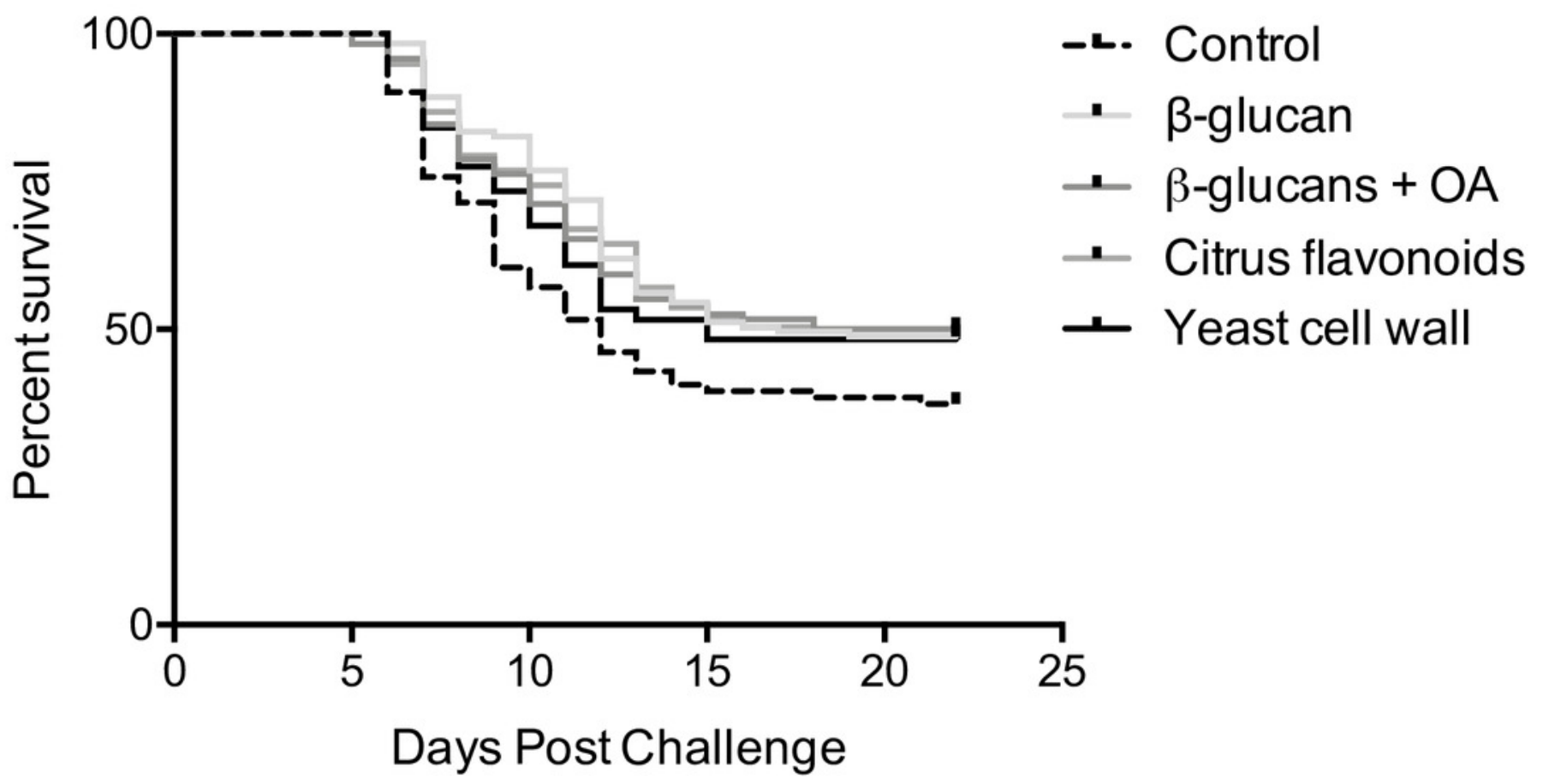


Figure 2

Hazard ratios relative to the control group

Hazard ratios relative to the control group, including $95 \% \mathrm{Cl}$. The vertical dotted line represents a hazard ratio of 1 , indicating no difference in relative risk. Statistical analysis is explained in Materials \& Methods.

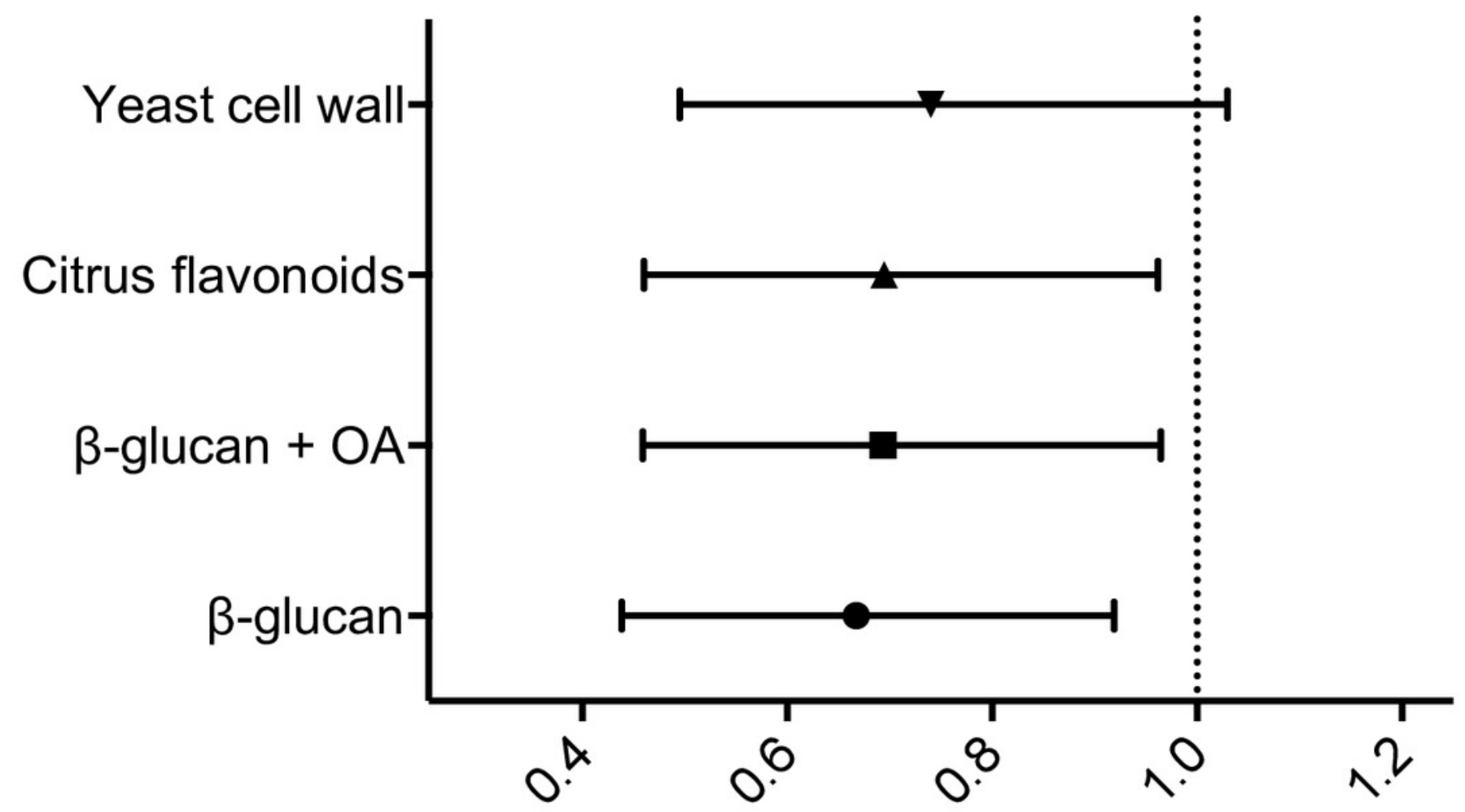

